\title{
ESTUDO DA VARIABILIDADE DE BILIRRUBINA TOTAL E DIRETA EXPOSTAS À LUZ AMBIENTE
}

\section{Amanda Vargas de FREITAS ${ }^{1}$, Melina Vargas de FREITAS ${ }^{1}$, Cristiano Guilherme Alves de OLIVEIRA ${ }^{1}$, Wilkes de OLIVEIRA ${ }^{2}$, Yuri Morais de Paula KASHIMA ${ }^{2}$, Juliano Gomes BARRETO ${ }^{1}$}

1 Universidade Iguaçu - Campus V. Itaperuna, Rio de Janeiro, Brasil.

2 Laboratório Kashima. Guaçuí, Espírito Santo, Brasil.

*Autor para correspondência: julianobarreto@hotmail.com

DOI: http://dx.doi.org/10.18571/acbm.187

\section{RESUMO}

A bilirrubina é um pigmento amarelo que é produzido pelo corpo quando as hemácias são degradadas. As amostras da referida substância devem ser protegidas da ação direta da luz artificial ou solar pois em presença dessas podem ser foto-oxidadas. O presente trabalho teve como objetivo avaliar a variabilidade das amostras de bilirrubina total e direta expostas à luz ambiente, o grau de porcentagem de diferença entre os resultados. Para melhor entender o seguinte trabalho foram obtidas amostras de forma aleatória, sendo estas coletadas em duplicata onde uma foi exposta diretamente à luz ambiente por período de $8 \mathrm{~h}$ e a outra protegida de exposição, sendo então os referidos tubos envolvidos em papel alumínio e abrigados em gaveta de madeira sob a bancada/armário do laboratório. Por meio dos resultados da referida pesquisa pôde-se observar uma diferença quanto à leitura das amostras, considerando o tempo e o modo de conservação destas.

Palavras-chave: Foto-oxidação; Bilirrubina; Luz ambiente.

\begin{abstract}
Bilirubin is a yellow pigment that is produced by the body when erythrocytes are degraded. Samples of this substance should be protected from the direct action of artificial or solar light because in the presence of these they may be photo-oxidized. The present study had as objective to evaluate the variability of the total and direct bilirubin samples exposed to light, the degree of percentage of difference between the results. To better understand the following work, random samples were obtained, and these were collected in duplicate where one was exposed directly to ambient light for a period of 8 hours and the other protected from exposure, and the said tubes were then wrapped in aluminum foil and housed in a drawer under the laboratory countertop / cabinet. By means of the results of the mentioned research a difference in the reading of the samples could be observed, considering the time and the way of conservation of these samples.
\end{abstract}

Keywords: Photo-oxidation; Bilirubin; Ambient light.

\section{Introdução}

A principal finalidade dos testes laboratoriais é auxiliar o médico no diagnóstico do paciente. Para isso, todas as fases dos testes devem ser conduzidas seguindo o rigor técnico necessário para a garantia da segurança e eficácia do resultado.

As fases da análise laboratorial são: pré-analítica, analítica e pós-analítica. A fase préanalítica concentra a maior parte dos erros no laboratório, estima-se que seja em torno de $70 \%$, entre eles está a orientação ao paciente dos exames que necessitam jejum, a alimentação, o uso de 
medicamentos capazes de interferir no resultado, a prática de exercício físico, entre outros. A fase analítica é a etapa de execução do teste, e por fim vem a fase pós analítica, que compreende a validação dos dados, e consequente liberação dos laudos para o médico prescritor fazer a avaliação do resultado (MASSAKAZU, 2014). A fase analítica segundo Teixeira (2016), é um processo de operações específico utilizado para a realização dos exames de acordo com métodos já conhecidos, esses erros podem variar de $7 \%$ a $13 \%$, sendo que eles podem derivar da troca de amostras ou à interferência e/ou mau funcionamento do equipamento.

Dentre os aspectos que necessitam de maior atenção, são estes: orientação ao paciente sobre o preparo correto para a coleta, identificação e transporte adequado do material biológico, informações relevantes como idade, raça, cor, sexo, medicamentos em uso, e processamento da amostra. Segundo Santos (2018), a falta de capacitação dos profissionais e a falta de informação dos pacientes durante a fase pré-analítica são grandes problemas, sendo necessário treinamento dos profissionais tanto para a coleta quanto no troca de informações e orientação aos pacientes. Apesar de ser difícil a eliminação total de erros já que se trata de tarefas manuais, o avanço das tecnologias e o surgimento da automação tem contribuído muito para minimização destes erros.

Segundo Rivello e Lourenço (2013) citam, de acordo com a Sociedade Brasileira de Patologia Clínica e Medicina Laboratorial, muitos dos erros nocivos acontecem particularmente no momento do preparo do paciente, por isso a importância de algumas informações serem coletadas.

Ulisses Tuma, 2010, em uma entrevista à revista Pharmacia Brasileira, comentou os problemas referentes à troca de amostras, que depende muitas vezes do setor de triagem do laboratório. Hoje em dia, o código de barras tornou mais segura esta questão. Disse que a SBAC tem se empenhado em seus Congressos, com palestras e cursos que orientam os profissionais sobre o assunto. Relatou que a RDC 302/05 ofereceu uma importante melhoria no que se trata à qualidade dos laboratórios de pesquisas clínicas, porém ainda há muitos erros por falta de uma fiscalização mais presente, em todo o Brasil (TUMA, 2010).

Para prevenir determinados erros na fase pré-analítica, quem deveria informar corretamente o paciente sobre como proceder na fase anterior à coleta deveria ser o médico assistente e/ou seus auxiliares, como por exemplo, o tempo de jejum, a dieta, suspensão de medicamentos que possam interferir nos resultados, reforçando a extrema relevância que tais procedimentos tem para se obter um resultado satisfatório (HAWKINS, 2012).

Os fatores pré-analíticos tem relação direta com os resultados da maioria dos testes laboratoriais, são eles: alterações em função do tempo, posição do paciente, gênero, jejum, dieta, álcool e fumo, exercícios físicos, gestação, medicamentos em uso, coleta, temperatura, hemólise, luz e infusão de líquidos e medicamentos.

Existem ainda entre os fatores pré-analíticos, as chamadas variáveis de coleta, em que observa a temperatura em que é conservado o material biológico, o tempo de garroteamento, o sangue colhido em local de acesso venoso com infusão de líquidos e medicamentos (MASSAKAZU, 2014).

Segundo Burtis \& Bruns (2016), a bilirrubina tem sua forma conjugada e não-conjugada, ambas as amostras precisam ser protegidas da ação direta da luz artificial ou solar, pois em presença de luz branca ou ultravioleta podem ser foto-oxidadas, e é aconselhável que essa amostra seja matinal e em jejum, para evitar lipemia. É importante que seu armazenamento seja no escuro e em baixas temperaturas, pois sua sensibilidade à luz é dependente da temperatura. Amostras mantidas em refrigerador permanecem estáveis por três dias. Quando as amostras são congeladas a $-70^{\circ} \mathrm{C}$ e no escuro, sua estabilidade mantém-se por três meses.

O ideal é uma urina recente, devido sua instabilidade quando exposta a luz e temperatura ambiente, e se for necessário que esse teste seja retardado, essa amostra precisará ser mantida em um refrigerador de $2^{\circ} \mathrm{C}$ a $8^{\circ} \mathrm{C}$ sem o alcance da luz. 
Henry (2012), cita ainda que uma vez no intestino, a bilirrubina é metabolizada pelas bactérias. Os produtos iniciais, urobilinogênios, são hidrossolúveis e podem ser reabsorvidos e sofrer circulação enteroepática. Aumento das concentrações plasmáticas ocorre com produção aumentada de bilirrubina, como na anemia hemolítica, ou com depuração reduzida no plasma, na hepatite ou na cirrose. $\mathrm{O}$ excesso de urobilinogênio pode então aparecer na urina, podendo ser detectados por testes em tiras reagentes, mas a determinação de urobilinogênio acrescenta muito pouco aos testes-padrão da função ou lesão hepática. Por fim, o urobilinogênio intestinal é transformado em pigmentos das fezes como a estercobilina, sua ausência produz fezes com coloração de argila, representando um sinal precoce de comprometimento do metabolismo da bilirrubina.

Dentre muitos distúrbios congênitos do metabolismo da bilirrubina, que pode se apresentar com icterícia ou bilirrubina sérica elevada, o mais comum é a síndrome de Gilbert, encontrada em até 3\% a 5\% da população, o qual está relacionado com a atividade reduzida da bilirrubina-UDPglicuroniltransferase I. Ao mesmo tempo em que alguns homens com esse distúrbio apresentavam uma percentagem significativa de bilurrubinemia, nenhuma das mulheres o fazia (HENRY, 2012).

Nesses pacientes com síndrome de Gilbert a bilirrubina total apresenta-se normalmente elevada em 2 até $3 \mathrm{mg} / \mathrm{dL}$, podendo aumentar em jejum, porém raramente excedem a $5 \mathrm{mg} / \mathrm{dL}$. Já a síndrome de Crigler-Najjar resulta da deficiência grave de bilirrubina-UDPglicuroniltransferase, sendo o tipo I a mais grave, onde não existe nenhuma atividade enzimática no fígado e os bebês afetados desenvolvem hiperbilirrubinemia grave não conjugada, levando a icterícia nuclear, disposição de bilirrubina no cérebro, afetando particularmente os gânglios basais (HENRY, 2012).

Outro distúrbio que envolve o comprometimento à excreção de bilirrubina conjugada, sem pigmentação hepática, é a síndrome de Rotor, porém sua causa ainda é desconhecida. Além das hepatopatias, outros distúrbios também podem provocar o aumento da bilirrubina, como a hemólise. Quando grave, pode ultrapassar a capacidade do fígado de captar a bilirrubina, com uma maior possibilidade de ocorrência em recém-nascidos, devido à baixa atividade da glicuroniltransferase (HENRY, 2012).

Septicemia, nutrição parenteral total e algumas drogas como androgênios geralmente causam aumento da bilirrubina conjugada, porém o mecanismo ainda é obscuro. Em indivíduos normais, o jejum provoca aumento da bilirrubina não conjugada, mas em grau menor do que aquele observado na síndrome de Gilbert (HENRY, 2012).

Com base na importância de detectar possíveis alterações em amostras devido a alguns fatores citados acima, temos por objetivo geral comparar as prováveis variações entre duas amostras de bilirrubina, uma exposta à luz ambiente e a outra protegida em tubo envolvido por papel alumínio.

\section{Materiais e Métodos}

O presente trabalho foi desenvolvido a partir de método de análise quantitativo, baseado em pesquisa experimental com produção de dados obtidos através de determinação de Bilirrubina Total e Direta em amostras de laboratório coletadas com finalidade diagnóstica para determinação de exames bioquímicos em geral.

As amostras foram obtidas de forma aleatória, sendo estas coletadas em duplicata onde uma foi exposta diretamente a luz ambiente por período de $8 \mathrm{~h}$ e a outra protegida de exposição à luz sendo então os referidos tubos envolvidos em papel alumínio e abrigados em gaveta de madeira sob a bancada/armário do laboratório. A identificação das amostras foi realizada de forma numérica e seqüencial conforme a coleta efetuada, seguidos respectivamente da identificação "com exposição" ou "sem exposição" para indicar qual amostra fora exposta à luz e qual fora 
protegida contra exposição, sendo suprimidos os dados de identificação de cada paciente afim de manter o sigilo profissional e ético no desenvolvimento deste trabalho.

A técnica empregada para realização analítica foi espectrofotometria enzimática de ponto final, utilizando o equipamento: BS 200, Marca: Kovalent®, e reagentes para a determinação de mesma marca.

A coleta das amostras ocorreu entre os meses de outubro/2017 e fevereiro/2018 sendo efetuada pelo próprio Laboratório, seguindo protocolo existente no mesmo, o qual foi responsável pela seleção aleatória das amostras, identificação correta das amostras, sigilo das informações e controle das variáveis pré e pós-analíticas.

Os dados foram tabulados em planilha Excel e tratados estatisticamente segundo a correlação matemática dos dados pelo método do percentual de queda frente a exposição e significância.

\section{Resultados e Discussão}

Os dados do presente trabalho são provenientes de 35 amostras de bilirrubina total e direta colhida aleatoriamente em um laboratório de analises clínicas. Foi analisada a variabilidade das amostras quando expostas a luz ambiente, por um período de 8 horas.

Pode-se observar na Tabela I que, 5 amostras de bilirrubina com valores entre 0,9 a 1,2 não alteraram com exposição à luz ambiente, e outras 5 obtiveram uma recaída de $5 \%$ em seus valores, quando expostas à luz.

Tabela 1: Amostras com resultados de bilirrubina total de 0,9 até 1,2.

\begin{tabular}{lcc}
\hline \multicolumn{1}{c}{ AMOSTRAS } & BIL TOTAL S/E (mg/dL) & BIL TOTAL C/E (mg/dL) \\
\hline Amostra 2 & 0,9 & 0,9 \\
Amostra 3 & 1,1 & 1,0 \\
Amostra 4 & 0,9 & 0,8 \\
Amostra 10 & 0,9 & 0,8 \\
Amostra 13 & 1,0 & 1,0 \\
Amostra 14 & 0,9 & 0,8 \\
Amostra 16 & 1,1 & 1,0 \\
Amostra 18 & 0,9 & 0,9 \\
Amostra 23 & 1,0 & 1,0 \\
Amostra 32 & 0,9 & 0,9 \\
\hline
\end{tabular}

Segundo Fischback (2002), citado por Tosta, Narciso, et al.; para dosagem de bilirrubina, os tubos com as amostras de sangue precisam ser protegidos da luz com papel alumínio ou então acondicionadas em frasco âmbar. Deve-se evitar a exposição a qualquer tipo de luz, artificial ou natural, durante o período pré-analítico.

Nota-se na Tabela 2, que todas as amostras com valores acima de 1,3 apresentaram uma recaída de $8,5 \%$ em seus valores, quando expostas à luz ambiente.

Tabela 2: Amostras com resultados de bilirrubina total acima de 1,3.

\begin{tabular}{lcc}
\multicolumn{1}{c}{ AMOSTRAS } & BIL TOTAL S/E (mg/dL) & BIL TOTAL C/E (mg/dL) \\
\hline Amostra 1 & 1,7 & 1,2 \\
Amostra 5 & 16,2 & 14,9 \\
Amostra 20 & 1,3 & 1,2 \\
Amostra 25 & 11,6 & 10,8 \\
Amostra 26 & 7,6 & 7,3 \\
Amostra 28 & 1,3 & 1,1 \\
Amostra 35 & 2,1 & 1,75 \\
\hline
\end{tabular}


A bilirrubina total pode diminuir até $50 \%$ em uma hora, sob exposição à luz solar direta (BECKMAN COULTER, 2018).

No tocante aos exames laboratoriais os interferentes podem ser analíticos, pré-analíticos ou pós-analíticos. Um dos exames que sofrem grande interferência pré-analítica é a bilirrubina direta que quando exposta a luz por determinado tempo sofre degradação com redução da concentração final. Desta forma, pode-se observar na figura 1 a variabilidade do perfil de bilirrubina direta separadas de acordo com a porcentagem de variação.

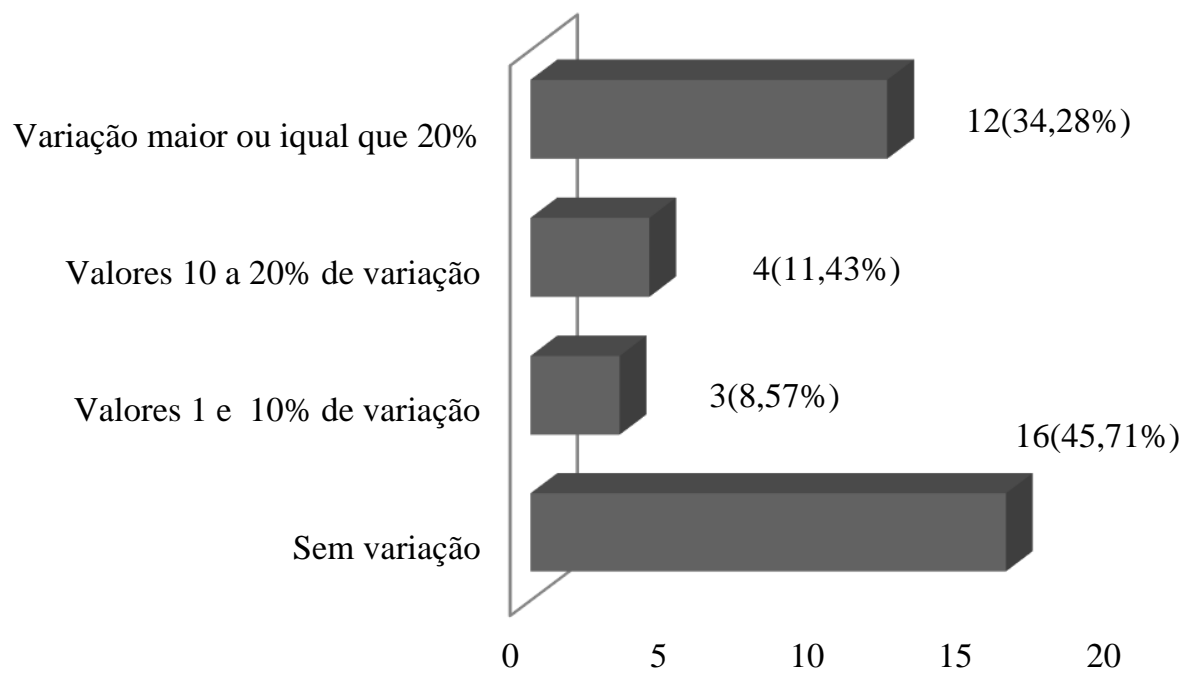

Figura 1: Porcentagem de variabilidade de bilirrubina direta.

Nota-se, no que se refere à variabilidade, que 16 amostras (ou seja 45,71\%) não sofreram alteração alguma quando expostas à luz, enquanto que 12 amostras (34,28\%) obtiveram variabilidade maior ou igual a 20\%, 4 amostras (11,43\%) com variação entre 10 a 20\%, e por fim 3 amostras $(8,57 \%)$ com valores entre 1 a $10 \%$ de variabilidade.

Observa-se que a maior parte das amostras sofreu redução em seus níveis de bilirrubina totalizando 54,28\% das amostras analisadas, contra um percentual de 45,71\% de amostras que não sofreram variação significativa nos níveis de bilirrubina avaliados, considerando aindaque,dentre as amostras que sofreram variabilidade na dosagem de bilirrubina, foi evidenciado que $34,28 \%$ destas sofreram uma redução maior ou igual a $20 \%$ nos níveis séricos de bilirrubina.

No que tange os resultados de bilirrubina total, a figura 2 abaixo demonstra a variabilidade das amostras, sendo distribuídas em valores de porcentagem. 


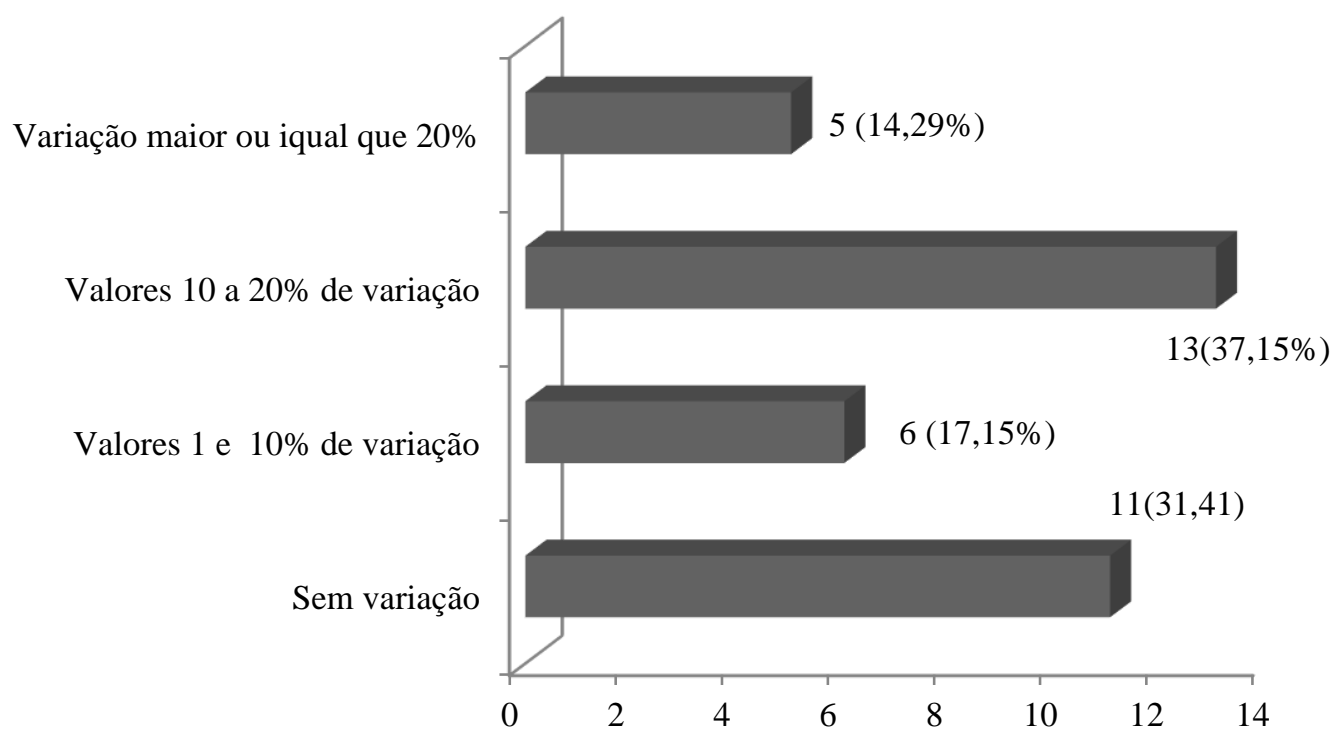

Figura 2: Porcentagem de variabilidade de bilirrubina total

Observa-se, em relação aos resultados da bilirrubina total, que 11 amostras (ou seja $31,41 \%)$ não sofreram alteração quando expostas à luz, enquanto que 13 amostras $(37,15 \%)$ obtiveram valores de variabilidade de $10 \%$ a $20 \%, 5$ amostras $(14,29 \%)$ com variação maior ou igual a $20 \%$, e por fim 6 amostras $(17,15 \%)$ com percentuais entre 1 a $10 \%$ de variabilidade.

Constata-se que a maior parte das amostras de bilirrubina total sofreu diminuição, totalizando o valor de $68,59 \%$ das amostras analisadas, contra um percentual de $31,41 \%$ de amostras que não sofreram nenhuma variação significativa nos níveis de bilirrubina analisados. Considera-se ainda que, dentre as amostras que sofreram variabilidade na dosagem de bilirrubina, foi demonstrado que $14,29 \%$ destas tiveram uma redução maior ou igual a $20 \%$ nos níveis séricos de bilirrubina.

No que se refere aos resultados de bilirrubina direta, a figura 3 a seguir apresenta a variabilidade das amostras em função dos valores de referência $(0,4 \mathrm{mg} / \mathrm{dL})$, sendo distribuídas em valores percentuais.

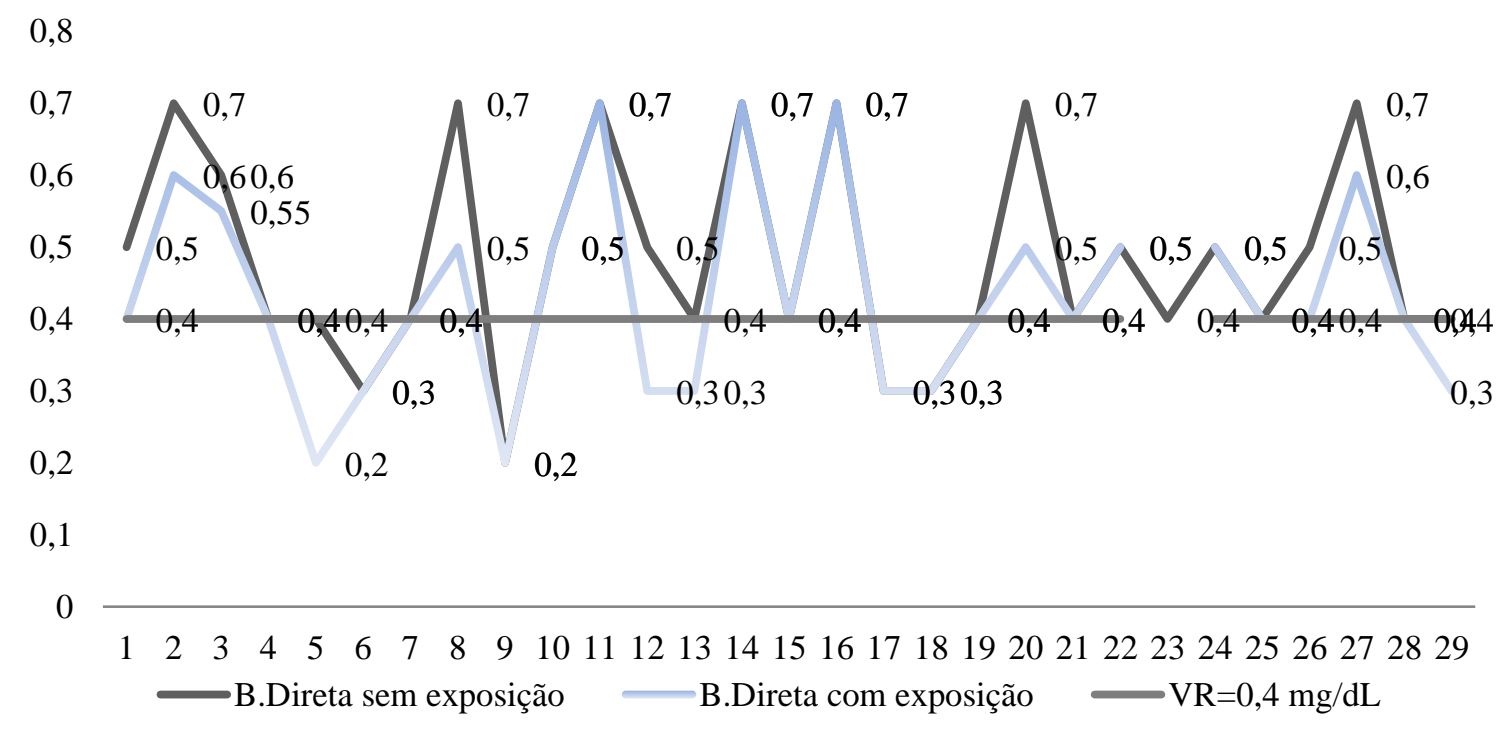

Figura 3: Variabilidade da bilirrubina direta em função dos valores de referência. 
Pode-se notar que, a bilirrubina direta sem exposição à luz obteve valores muito acima do valor de referência citado no gráfico. Já as amostras com exposição à luz por um período de 8 horas alcançaram valores abaixo do esperado.

Para o estudo da variabilidade em função do valor de referência de bilirrubina total tem-se os seguintes resultados:

Observa-se, na figura 4, a análise feita de 32 amostras de bilirrubina total, 4 amostras sem exposição obtiveram valores acima do valor de referência, sendo 2 com expressivo resultado, $1,7 \mathrm{mg} / \mathrm{dL}$ e $2,1 \mathrm{mg} / \mathrm{dL}$, respectivamente. Em seguida, apresenta-se apenas 1 amostra com exposição acima do valor de referência, todas as outras foram abaixo do referido valor.

Para a realização dos gráficos foram retiradas 5 amostras com valores muito acima do valor de referência da bilirrubina, para uma melhor visualização dos mesmos e pelo motivo de que com valores muito acima do normal, esses mesmos valores quando expostos a luz sofreram uma queda, porém não o suficiente que possa ter relevância no diagnóstico final, continuando com seus valores acima do valor normal da bilirrubina. Já os valores mais baixos e próximos do valor de referência $(1,2)$, quando expostos a luz também obtiveram uma queda, e essa queda resultou em um valor normal ou abaixo do de referência, podendo assim resultar em um diagnóstico falso-positivo.

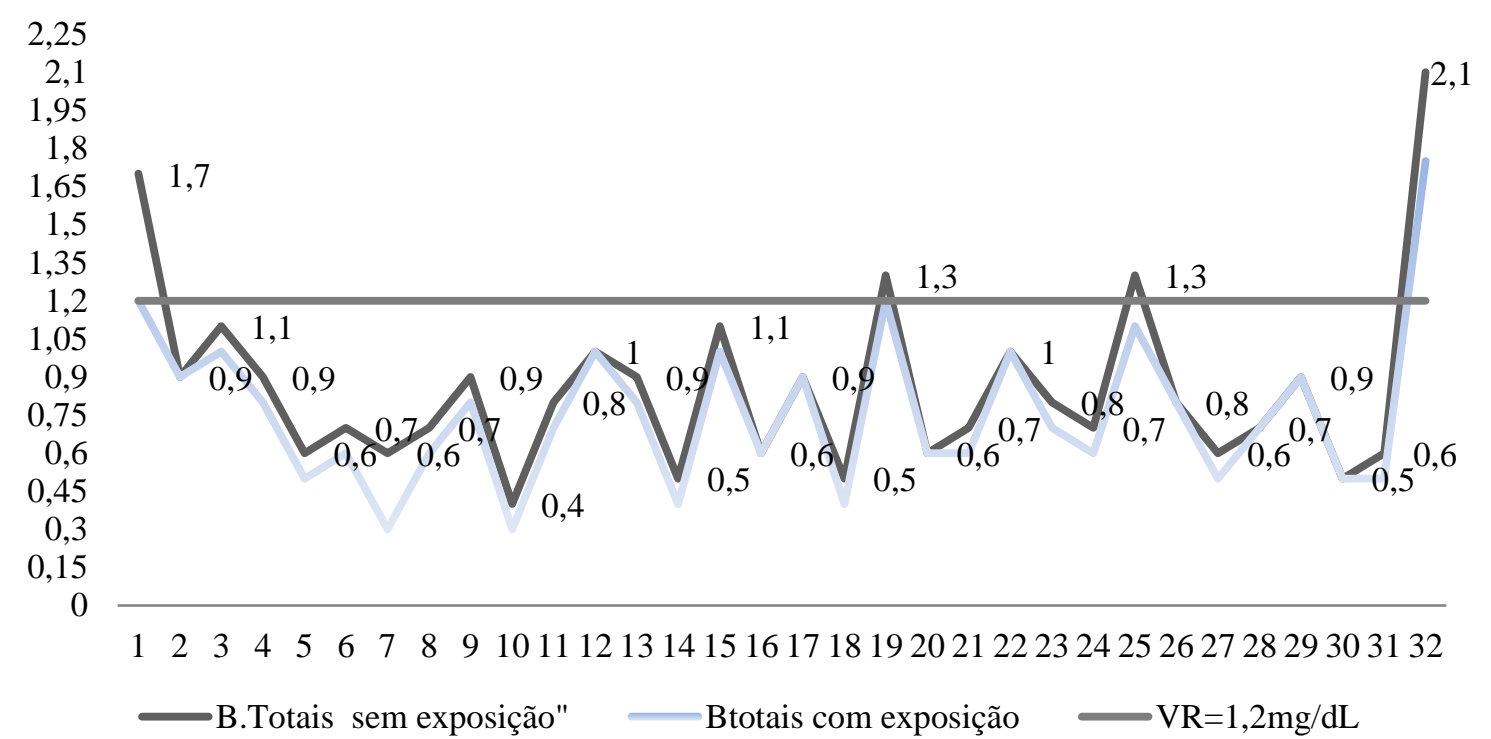

Figura 4: Variabilidade de bilirrubina total em função dos valores de referência.

\section{Conclusão}

Conclui-se, portanto, que a fase pré-analítica tanto na bilirrubina total como a direta é de total importância para um resultado correto e eficaz, onde na pesquisa observamos que a maioria das amostras sofreram redução em seus níveis séricos de bilirrubina quando expostas à luz, e que seu armazenamento e conservação inapropriados acarreta alterações significativas no resultado final, podendo assim ocasionar resultados falsamente diminuídos e/ou diagnóstico e tratamento falho e ineficaz.

Tosta, et al (2011), aponta para a dificuldade em relacionar o presente trabalho com resultados anteriores, considerando que encontram-se poucos trabalhos publicados neste sentido, com a finalidade de avaliar a fotossensibilidade da bilirrubina, o que sugere a necessidade de novos e mais amplos estudos com este objetivo de melhor esclarecer os níveis e percentuais de comprometimento da exposição luminosa de amostras séricas destinadas a dosagem de bilirrubina. 


\section{Referências}

BECKMAN COULTER. Instruções de uso: Bilirrubina direta. São Paulo: Beckman Coulter do Brasil, 2018.

BURTIS, C. A.; BRUNS, D. E. Tietz Fundamentos de Química Clínica e Diagnóstico Molecular. 7. ed. Rio de Janeiro: Guanabara Koogan, 2016.

HAWKINS, Robert. Managing the Pre- and Post-analytical Phases of the Total Testing Process. Ann Lab Med. 2012 Jan; 32(1): 5-16.

HENRY, J. B. Diagnósticos clínicos e tratamento por métodos laboratoriais. 21. ed. São Paulo: Manole, 2012.

MASSAKAZU SUMITA, Nairo. Exames laboratoriais e a importância dos cuidados préanalíticos. Disponível em:< http://www.fleury.com.br/medicos/educacaomedica/artigos/Pages/exames-laboratoriais-e-a-importancia-dos-cuidados-pre-analiticos.aspx > Acesso em: 28 de novembro de 2017.

RIVELLO, V.V.; LOURENÇO, P.M. A prevalência de erro na fase pré-analítica nos laboratórios de análises clínicas. Revista Saúde. 2013 Jan./Dez

SANTOS, C. N.; PÁUDA, P. D.; SANTOS, R. W. F.; SANTOS, R. C. F.; ALMEIDA JR, E.; REIS, F. P. Exames laboratoriais e a fase pré-analítica. SEMPESQ. 18ed, UNIT. Aracajú, SE 2018.

TEIXEIRA, J. C. C.; CHICOTE, S. R. M.; DANEZE, E. R. Não conformidades identificadas durante as fases pré-analítica, analítica e pós-analítica de um laboratório público de análises clínicas. Nucleus, v. 13, n. 1, p. 251-260, 2016.

TOSTA, Thaísa. et al. Influência da luz na determinação da bilirrubina total em bovinos. $38^{\circ}$ Congresso Brasileiro de Medicina Veterinária (Conbravet). Florianópolis, SC. Nov/ 2011.

TUMA, Ulisses. Análises Clínicas: Controle de qualidade na fase pré-analítica. RevistaPharmacia Brasileira, Março/Abril.2010. 